\title{
Polygenic Risk Scores for Developmental Disorders, Neuromotor Functioning During Infancy, and Autistic Traits in Childhood
}

\author{
Fadila Serdarevic, Henning Tiemeier, Philip R. Jansen, Silvia Alemany, Yllza Xerxa, \\ Alexander Neumann, Elise Robinson, Manon H.J. Hillegers, Frank C. Verhulst, and \\ Akhgar Ghassabian
}

\begin{abstract}
BACKGROUND: Impaired neuromotor development is often one of the earliest observations in children with autism spectrum disorder (ASD). We investigated whether a genetic predisposition to developmental disorders was associated with nonoptimal neuromotor development during infancy and examined the genetic correlation between nonoptimal neuromotor development and autistic traits in the general population.

METHODS: In a population-based cohort in The Netherlands (2002-2006), we calculated polygenic risk scores (PRSs) for ASD and attention-deficit/hyperactivity disorder (ADHD) using genome-wide association study summary statistics. In 1921 children with genetic data, parents rated autistic traits at 6 years of age. Among them, 1174 children $(61.1 \%)$ underwent neuromotor examinations (tone, responses, senses, and other observations) during infancy (9-20 weeks of age). We used linear regressions to examine associations of PRSs with neuromotor scores and autistic traits. We performed a bivariate genome-based restricted maximum likelihood analysis to explore whether genetic susceptibility underlies the association between neuromotor development and autistic traits.

RESULTS: Higher PRSs for ASD were associated with less optimal overall infant neuromotor development, in particular low muscle tone. Higher PRSs for ADHD were associated with less optimal senses. PRSs for ASD and those for ADHD both were associated with autistic traits. The single nucleotide polymorphism-based heritability of overall motor development was $20 \%(\mathrm{SE}=.21$ ) and of autistic traits was $68 \%(\mathrm{SE}=.26)$. The genetic correlation between overall motor development and autistic traits was .35 (SE $=.21, p<.001)$.

CONCLUSIONS: We found that genetic liabilities for ASD and ADHD covary with neuromotor development during infancy. Shared genetic liability might partly explain the association between nonoptimal neuromotor development during infancy and autistic traits in childhood.
\end{abstract}

Keywords: ADHD, Autism, Cohort, Infant, Neuromotor, Polygenic risk score, Population based

https://doi.org/10.1016/j.biopsych.2019.06.006

Autism spectrum disorder (ASD) is a lifelong developmental disorder characterized by social interaction impairment, communication deficits, and repetitive behavior. Evidence confirms that autistic traits, defined as social deficits that do not meet the formal criteria for an ASD diagnosis, have a continuous distribution in the general population (1). ASD has a strong genetic basis and shares genetic risks with other heritable disorders of childhood, for example, attention-deficit/ hyperactivity disorder (ADHD) (2). Similar to ASD, autistic traits show moderate to high heritability in the general population as well as in the clinical samples (3).

Behavioral signs of ASD typically emerge mostly during the second year of life, and the few symptoms that occur during the first year of life mainly belong to the sensorimotor domain (4). Nonoptimal neuromotor development is often one of the earliest identifiable clinical observations in children with ASD
(5). The motor manifestations encompass a wide range of impairments, including floppiness, delays in gross motor milestones such as sitting, and poor motor coordination and control, for example, difficulties in grasping objects. Many studies have reported co-occurrence of motor impairments with autistic traits and autism $(6,7)$, and follow-up studies have shown early sensorimotor manifestations before an autism diagnosis in high-risk infants $(8,9)$. We have previously shown that neuromotor development measured during infancy was associated with autistic traits in the current study population (10). Neuromotor development during infancy can be a measure of overall neurodevelopmental delay. However, it is not a general measure of higher brain functions because nonoptimal neuromotor development during infancy is not associated with nonverbal intelligence in children (11). While impaired neuromotor development can be a symptom of an underlying brain 
abnormality related to autistic symptoms, inflexible sensorimotor development during infancy is also posited to precede an abnormal developmental trajectory of the brain and subsequently autism $(12,13)$. Studies have suggested that infant sensorimotor variations are associated with increased risk of autistic symptoms, with recent evidence from follow-up and twin studies suggesting that sensorimotor problems may lead to autistic symptoms $(12,14)$. However, the possible genetic contribution to this association is unknown. Common genetic risk or antecedent environmental factors might explain the observed association between nonoptimal neuromotor development and autistic traits. If genetic variations for neurodevelopmental disorders are related to early motor abnormalities, the latter can be used as a risk indicator to explore the susceptibility of infants to autism.

Accordingly, the aim of this study was to investigate the associations between a genetic predisposition to neurodevelopmental disorders and neuromotor functioning during infancy and explore whether infant neuromotor functioning shares a genetic liability with autistic traits in the general population. We examined the association of polygenic risk

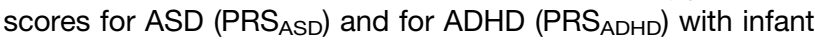
neuromotor development because it is less clear whether minor neuromotor deficits are specifically related to genetic predisposition to ASD or constitute a broader reflection of genetic susceptibility to neurodevelopmental disorders in general. We further investigated whether there is pleiotropy between infant neuromotor development and autistic traits due to genetic liability.

\section{METHODS AND MATERIALS}

This study was embedded in the Generation R Study, a population-based birth cohort in Rotterdam, The Netherlands, which recruited more than 9000 pregnant women with a delivery date from April 2002 to January 2006 to study early determinants of development and health in childhood and adolescence (15). From this birth cohort, we included a pediatric sample of European ancestry with available genotype data $(n=2830)$ (16). Between 9 and 20 weeks of age, 1174 infants $(41 \%$ of 2830 with genotyping data) underwent a neuromotor examination during home visits. When the children were 6 years old, questionnaires were mailed to caregivers for assessments of autistic traits ( $n=1921$ children, $68 \%$ of 2830) (Supplemental Figure S1).

We compared child and maternal characteristics of the children included in this analysis $(n=1921)$ with those excluded because of missing data on autistic traits $(n=909)$. Children included in the analyses were more likely to have higher nonverbal IQ scores than children excluded (mean IQ scores $=103.1$ vs. 96.4). Participating mothers also had lower scores of psychopathology symptoms during pregnancy compared with nonparticipating mothers (mean scores based on the Brief Symptoms Inventory $=0.24$ vs. 0.37). However, two groups of children did not differ on neuromotor assessments during infancy. In addition, children included in the analysis did not differ on autistic traits compared with children excluded due to missing data on genetic assessments.

The study protocol was approved by the Medical Ethics Committee of the Erasmus Medical Center. Written informed consent was obtained from the legal representatives of all participants.

\section{Genotyping and Imputation}

DNA samples were collected from the cord blood or venipuncture at 6 years of age on Illumina 610,000 - and 660,000single nucleotide polymorphism (SNP) arrays depending on collection time (Illumina, San Diego, CA). Others have previously reported further details on genotype calling procedures in Generation R (17). Quality control of the genotype- and individual-level data was conducted using PLINK (version 1.9) (18). Genotype data quality control included filtering variants for minor allele frequency $(<0.01)$, Hardy-Weinberg disequilibrium $\left(p<1 \times 10^{-5}\right)$, and missing rate $(>0.05)$. Individuals were excluded according to relatedness, genetic and phenotypic sex mismatch, and genotype quality ( $<5 \%$ missing).

Imputation was carried out to the 1000 genomes (phase I version 3) using prephasing in SHAPEIT (19) and imputation in IMPUTE (version 2) (20). Postimputation filtering on imputation quality (info score $<0.9$ ) resulted in a total of $6,561,671$ variants. To correct for population stratification, we calculated four genetic principal components using principal component analysis in EIGENSOFT and included them in regression models $(21,22)$.

\section{Polygenic Scoring}

We used imputed genotype data that passed quality control to compute PRSs based on genome-wide association studies (GWASs) of ASD and ADHD performed by the Psychiatric Genomics Consortium. PRS $_{\mathrm{ASD}}$ and $\mathrm{PRS}_{\mathrm{ADHD}}$ were created using PRSice (23). This software calculates individual PRSs by summing up all the SNP alleles associated with the trait carried by the participants weighted by the SNP allele effect size estimated in a previous GWAS. Polygenic scoring was performed in clumped variants according to linkage disequilibrium using an $r^{2}<.10$ cutoff within a $250-\mathrm{kb}$ window. We calculated PRSs for each trait based on six different $p$-value thresholds $\left(p_{\mathrm{T}} \mathrm{s}\right)$ with $p_{\mathrm{T}}<.001, p_{\mathrm{T}}<.01, p_{\mathrm{T}}<.05, p_{\mathrm{T}}<.10, p_{\mathrm{T}}<.50$, and $p_{\mathrm{T}}<1$. Supplemental Table S1 presents the number of variants included in the PRSs for each $p_{\mathrm{T}}$. All PRSs were standardized to a mean of 0 and a standard deviation of 1 .

\section{Infant Neuromotor Development}

During home visits, trained research assistants carried out full neuromotor assessments for infants aged 9 to 20 weeks using an age-appropriate and modified version of the Touwen's Neurodevelopmental Examination (Supplemental Table S2) (24). We used two age-adapted versions of the neuromotor instrument for 9 to 15 weeks and 16 to 20 weeks; both versions encompassed assessments of tone, responses, senses, and other observations (e.g., spontaneous movements) (25). Tone was assessed in several positions - supine, horizontal, vertical, prone, and sitting - and all tone items, such as adductor angle, were scored as normal, low, or high tone. Following the approach described by de Groot et al., we used an adapted version of Touwen including items measuring both active muscle strength and passive muscle tone (25). Measures of both active muscle strength and passive muscle tone can be early signs of deviation from typical motor development. Within 
the subscale measuring tone, a further distinction was made between low tone and high tone, resulting in two additional subscales for tone: low muscle tone and high muscle tone. Responses were assessed in vertical, supine, or prone position and were scored as present, absent, or excessive. Senses and other observations comprise strabismus, fixation eyes, following movement eyes, hearing, sweating, and startled reactions (26). The later items were scored as present, absent, or excessive. For each item, we labeled an age-appropriate response as optimal. If the response indicated a delayed development, the item was labeled nonoptimal. By summing the raw values of all items, we obtained a total score, for which high values indicated less optimal neuromotor development. All scores were used as continuous variables after square root transformation for normality. The intraclass correlation coefficients for the short-interval test-retest reliability and the interobserver reliability were .52 and .64 , respectively, similar to earlier reports (27). We present the correlations between different neuromotor outcomes in Supplemental Table S3.

\section{Autistic Traits}

When the children were 6 years old, we administered the Social Responsiveness Scale (SRS) to parents to obtain a quantitative measure of children's autistic traits during the past 6 months in a naturalistic setting (1). Due to the length of the original questionnaire, we used a short version of the SRS with 18 items. The 18-item questionnaire contained items concerning social cognition, social communication, and autistic mannerism. In Generation R, the Cronbach's alpha indicated high interitem reliability for the SRS $(\alpha=.79)$. In a sample of 3857 children aged 4 to 18 years in the South-West of The Netherlands (2010-2012), the correlation between total scores derived from the selected 18 items and the scores derived from the complete test was $r=.95$. Each item is rated from 0 (never true) to 3 (almost always true). SRS scales were used continuously after square root transformation and standardization.

\section{Covariates}

We used medical records completed by community midwives and obstetricians to obtain information on the children's sex. Information on maternal age and educational levels was obtained from self-reported questionnaires. Maternal education was defined by the highest attained educational level and was classified as primary (no or only primary education), secondary (lower or intermediate vocational education), or higher education (higher vocational education or university).

\section{Statistical Analysis}

We performed linear regression analyses to examine whether $\mathrm{PRS}_{\mathrm{ASD}}$ and $\mathrm{PRS}_{\mathrm{ADHD}}$ were associated with infant neuromotor scores (overall neuromotor development including the following subscales: low muscle tone, high muscle tone, responses, and senses and other observations) and autistic traits at 6 years of age. Genetic risk scores of ADHD and ASD could potentially be correlated. Therefore, in an additional step, we also mutually adjusted our models for $\mathrm{PRS}_{\mathrm{ASD}}$ and $\mathrm{PRS}_{\mathrm{ADHD}}$ to test unique effects of each PRS on outcomes (16). Based on an a priori hypothesis, we also studied whether the magnitude of the effect depended on sex by formally testing interaction with sex $(28,29)$. We performed correction for multiple comparisons using Bonferroni adjustment (10 hypotheses with 5 independent outcomes [low muscle tone, high muscle tone, responses, senses and other observations, and autistic traits] and 2 exposures [PRS ADHD $_{\text {and }}$ PRS $\left._{\mathrm{ASD}}\right] ; \alpha=.05 / 10=.005$ ).

We previously showed associations between infant muscle tone and autistic traits (10). Next, we explored whether the shared genetic susceptibility could underlie the observed association between neuromotor development and autistic traits. We performed a bivariate genome-based restricted maximum likelihood analysis to examine the shared liability of overall neuromotor development (as well as overall tone) and autistic traits by genetic predispositions (30). This analysis provides the SNP-based heritability associated with neuromotor development and autistic traits as well as the genetic correlation between two outcomes. We calculated a genetic relatedness matrix based on 504,617 autosomal SNPs. All SNPs were directly genotyped and had a minor allele frequency higher than $1 \%$. We then estimated to what extent the similarity in the genotype between participants can explain the similarity in neuromotor development and autistic trait scores among participants as well the extent to which the genetic relatedness matrix can explain the covariance of both traits. The resulting estimate of genetic correlation indicates the correlation between motor development and autism traits that is due to shared genetics. The genome-based restricted maximum likelihood analyses were performed in GCTA 1.26.0 (31).

In a sensitivity analysis, we explored whether additional adjustment for PRSs for schizophrenia would change our findings on $\mathrm{PRS}_{\mathrm{ASD}}$ and $\mathrm{PRS}_{\mathrm{ADHD}}$ (16). In this sample, 9 children had the confirmed diagnosis of ASD. We reran the analysis excluding ASD cases.

All analyses were performed using the R statistical software package (version 3.3.1)

\section{RESULTS}

Children had an average neuromotor score of 1.67 (SD $=0.96$ ), mean age at neuromotor assessment was 12.6 weeks (SD = 20 ), and $48.7 \%$ of children were girls. Mothers were on average 31.3 years old $(S D=4.7)$, and $55 \%$ of them completed higher education.

A higher $\mathrm{PRS}_{\mathrm{ASD}}$ was positively associated with less optimal overall neuromotor development during infancy (e.g., with GWAS $p_{\mathrm{T}}<1, \beta=.048,95 \%$ confidence interval $[\mathrm{Cl}]=.007$, $.090, p=.02$ ) (Figure 1 and Table 1). There was a relationship between $\mathrm{PRS}_{\mathrm{ASD}}$ and overall muscle tone and, in particular, low muscle tone (e.g., with GWAS $p_{\mathrm{T}}<.50, \beta=.068,95 \% \mathrm{Cl}=$ $.015, .120, p=.01)$. Stratified analysis showed that this association was present in boys only (Supplemental Table S4). In addition, boys with a higher PRS $_{\mathrm{ASD}}$ had less optimal scores in senses and other observations during infancy (e.g., with GWAS $\left.p_{\mathrm{T}}<.043, \beta=.003,95 \% \mathrm{Cl}=.003, .084, p=.02\right)$. A higher $\mathrm{PRS}_{\mathrm{ADHD}}$ was associated with less optimal senses and other observations $\left(p_{\mathrm{T}}<.01, \beta=.035, \mathrm{Cl}=.006, .065, p=.02\right)$, in particular in boys (e.g., with GWAS $p_{\mathrm{T}}<.01, \beta=.057,95 \%$ $\mathrm{Cl}=.015, .099, p=.01)$ (Supplemental Table S5). There was no association between $\mathrm{PRS}_{\mathrm{ASD}}$ or $\mathrm{PRS}_{\mathrm{ADHD}}$ and high muscle tone or responses (Figure 1 and Table 1). Results for 


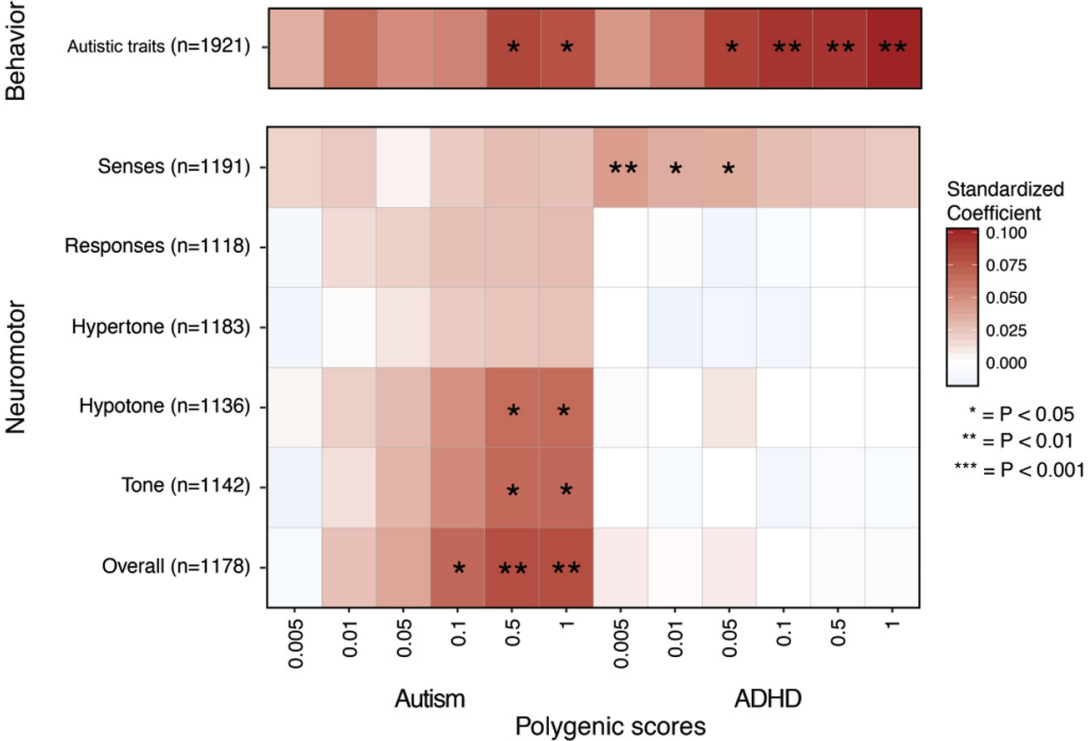

Figure 1. Associations of polygenic risk scores for autism spectrum disorder and for attention-deficit/ hyperactivity disorder (ADHD) with neuromotor development scores during infancy and autistic traits at 6 years of age. Effect sizes were estimated using linear regression models with neuromotor development and autistic traits as outcomes. Polygenic risk scores for autism spectrum disorder and for ADHD were selected based on different $p$-value thresholds $\left(p_{\mathrm{T}}\right): p_{\mathrm{T}}<.005, p_{\mathrm{T}}<.01, p_{\mathrm{T}}<.05, p_{\mathrm{T}}<.10, p_{\mathrm{T}}<.50$ and $p_{\mathrm{T}}<1$. Models were adjusted for a child's sex and age at assessment and four genetic principal components (and for the version of the neuromotor instrument in models with neuromotor development). associations of $\mathrm{PRS}_{\mathrm{ASD}}$ and $\mathrm{PRS}_{\mathrm{ADHD}}$ with overall neuromotor development remained after Bonferroni adjustment.

Children with higher $\mathrm{PRS}_{\mathrm{ASD}}$ had higher autistic trait scores at 6 years of age $\left(p_{\mathrm{T}}<.50, \beta=.08,95 \% \mathrm{Cl}=.03, .13, p=.01\right)$, with no interaction with sex (interaction $p=.91$ ) (Figure 1). In contrast, we found that the association between $\mathrm{PRS}_{\mathrm{ADHD}}$ and autistic traits at age 6 depended on sex (interaction $p<.03$ ). Boys, but not girls, with a higher genetic liability for ADHD had higher autistic trait scores $\left(p_{\mathrm{T}}<.10, \beta=.176,95 \% \mathrm{Cl}=.090\right.$,
.266, $p \leq .001$ ) (Supplemental Table S5 and Supplemental Figure S2). Results for $\mathrm{PRS}_{\mathrm{ADHD}}$ and the SRS remained after Bonferroni adjustment.

When we mutually adjusted our models for PRS $_{\text {ASD }}$ and $\mathrm{PRS}_{\mathrm{ADHD}}$, results did not materially change. Similarly, further adjustment for PRSs for schizophrenia did not influence the results (data not shown). The SNP-based heritability of overall motor development was $20 \%$ (SE = .21) and of autistic traits was $68 \%(\mathrm{SE}=.26)$. The genetic correlation between overall

Table 1. Associations Between Polygenic Risk Scores for Autism Spectrum Disorder and for Attention-Deficit/Hyperactivity Disorder With Infant Neuromotor Development and Autistic Traits

\begin{tabular}{|c|c|c|c|c|c|c|c|c|c|c|c|c|c|c|c|c|}
\hline & \multicolumn{4}{|c|}{$p_{\mathrm{T}}<.001$} & \multicolumn{4}{|c|}{$p_{\mathrm{T}}<.01$} & \multicolumn{4}{|c|}{$p_{\mathrm{T}}<.10$} & \multicolumn{4}{|c|}{$P_{\mathrm{T}}<1$} \\
\hline & $\beta$ & $95 \% \mathrm{Cl}$ & $p$ & $R^{2}$ & $\beta$ & $95 \% \mathrm{Cl}$ & $p$ & $R^{2}$ & $\beta$ & $95 \% \mathrm{Cl}$ & $p$ & $R^{2}$ & $\beta$ & $5 \% \mathrm{Cl}$ & $p$ & $R^{2}$ \\
\hline \multicolumn{17}{|c|}{ Polygenic Risk Scores for Autism Spectrum Disorder } \\
\hline vera & -.006 & $-.059, .046$ & .81 & .09 & .027 & $026, .080$ & .32 & .09 & .067 & $.015, .120$ & .01 & .10 & .081 & $.028, .134$ & .003 & .10 \\
\hline verall tone & -.009 & $-.062, .043$ & .73 & .09 & .013 & $-.039, .066$ & 62 & .08 & .052 & $-.001, .104$ & .05 & .08 & .068 & $.015, .120$ & .01 & .08 \\
\hline$w r$ & -.011 & $-.061, .039$ & .67 & .12 & .020 & $-.030, .071$ & .43 & .12 & .048 & $-.002, .099$ & .06 & .13 & .066 & $.016, .116$ & .01 & .13 \\
\hline gh $\mathrm{m}$ & .017 & $-.020, .054$ & .37 & .01 & .002 & $-.035, .039$ & .90 & .01 & .021 & $-.016, .058$ & .27 & .01 & .025 & $-.012, .062$ & .19 & .01 \\
\hline spc & .004 & $-.027, .034$ & .81 & .14 & .015 & $-.015, .045$ & .33 & .14 & .026 & $-.004, .057$ & .09 & .14 & .028 & $-.002, .059$ & .07 & .14 \\
\hline ense & .004 & -.024 & .76 & .01 & .022 & -.00 & .1 & .0 & .021 & -.00 & .1 & .0 & .027 & $-.002, .055$ & .06 & .01 \\
\hline utistic traits & -.011 & $-.077, .055$ & .75 & .02 & .065 & $-.001, .130$ & .05 & .03 & .055 & $-.011, .121$ & .10 & .03 & .079 & $.013, .015$ & .02 & .03 \\
\hline \multicolumn{17}{|c|}{ Polygenic Risk Scores for Attention-Deficit/Hyperactivity Disorder } \\
\hline erall neuromotor & .027 & $-.028, .082$ & .33 & .09 & .004 & $-.052, .059$ & .00 & .09 & -.002 & $-.057, .052$ & .30 & .00 & .002 & $-.051, .056$ & .94 & .00 \\
\hline erall tone & .019 & $-.034, .073$ & .48 & .08 & -.008 & $-.063, .046$ & .76 & .08 & -.013 & $-.066, .041$ & .64 & .08 & -.007 & $-.060, .046$ & .80 & .08 \\
\hline ow muscle tone & .030 & $-.022, .081$ & .26 & .12 & .001 & $-.052, .053$ & .98 & .12 & .001 & $-.051, .052$ & .98 & .12 & -.001 & $-.052, .050$ & .98 & .12 \\
\hline cle tone & -.002 & $-.040, .036$ & .92 & .01 & -.015 & $-.054, .024$ & .44 & .01 & -.013 & $-.051, .025$ & .51 & .01 & -.002 & $-.040, .035$ & .91 & .01 \\
\hline sponses & -.001 & $-.032, .031$ & .97 & .14 & .002 & $-.030, .034$ & .90 & .14 & -.007 & $-.038, .024$ & .67 & .11 & .000 & $-.031, .030$ & .99 & 14 \\
\hline enses & .043 & $.001, .060$ & .04 & .01 & .035 & $.006, .065$ & .02 & .02 & .028 & $-.001, .057$ & .06 & .01 & .022 & $-.006, .051$ & .12 & .01 \\
\hline utistic traits & .094 & $.029, .160$ & .01 & .03 & .098 & $.031, .164$ & .004 & .03 & .098 & $.032, .164$ & .004 & .03 & .111 & $.047, .176$ & .001 & .03 \\
\hline
\end{tabular}

Models were adjusted for children's sex and age at assessment and four genetic principal components (and for the version of the neuromotor instrument in models with neuromotor development).

$\mathrm{Cl}$, confidence interval; $p_{\mathrm{T}}$, significance threshold for inclusion of variants in the polygenic score. 
Infant Neuromotor, Autistic Traits, and PRSs

motor development and autistic traits was .35 (SE $=.21, p<$ .001), rejecting the null hypothesis on no genetic correlation between overall motor development and autistic traits. When we ran the analysis with overall tone, we found that the SNPbased heritability of overall tone was $24.3 \%(\mathrm{SE}=.21)$ and of autistic traits was $65 \%$ (SE = .26). The genetic correlation between overall tone and autistic traits was .32 (SE $=.17, p<$ .0002). Exclusion of the ASD cases from the analytical sample did not materially change the findings.

\section{DISCUSSION}

In this population-based study of children from European ancestry, a higher genetic liability for ASD was associated with less optimal overall infant neuromotor development and low muscle tone at 9 to 20 weeks of age. Genetic susceptibility contributes to both less optimal infant neuromotor development and autistic traits at 6 years of age. Both ASD and ADHD genetic susceptibility were related to less optimal senses and other observations and autistic traits in boys only.

Previous studies have shown associations of $P_{R S} S_{A S D}$ and $\mathrm{PRS}_{\mathrm{ADHD}}$ with neuropsychiatric symptoms in the general population $(32,33)$. Using large ASD consortia and populationbased resources $(n>38,000)$, Robinson et al. found that the genetic risk for ASD influences a continuum of sociobehavioral and developmental traits in the general population; the extreme of these traits comprises children with ASD or other neuropsychiatric disorders $(3,33)$. In the Avon Longitudinal Study of Parents and Children, Martin et al. reported associations between $\mathrm{PRS}_{\mathrm{ADHD}}$ and ADHD symptoms and reported pragmatic language abilities but not with social cognition (32). Riglin et al. examined the association between PRS $_{\text {ADHD }}$ and trajectories of symptoms for ADHD childhood and showed associations with autistic traits (34). In line with these earlier studies, we found in this study that PRS $_{A S D}$ and $P R S_{A D H D}$ were associated with autistic traits in children from the general population. Moreover, our study extends these findings to infants from the general population, showing that $P_{R S_{A S D}}$ and $\mathrm{PRS}_{\mathrm{ADHD}}$ predicted neuromotor development at a very early age before the emergence of social responsiveness problems. Early delays in motor development are a common feature in children with autistic symptoms (10), ASD (35), and ADHD (36). In a cohort of 114 children with ASD and their siblings, cooccurrence of motor impairment and attention problems predicted high autistic symptoms and diagnosis of ASD in the siblings of children with ASD (14). Typically, motor impairment and attention problems jointly appear before ASD is diagnosed, accounting for more than $50 \%$ of the autistic symptom variation in siblings of children with ASD. In this sample, motor proficiency score was the most important predictor of autistic symptoms and ASD diagnostic status in siblings of children with ASD (14). Yet, it has remained unclear why motor impairment tracked so closely with attention and social communication problems and what defines the cascade of impairments from genetic predisposition to early symptoms during infancy and later in childhood. While motor impairments can be a comorbidity of ASD, alternatively it can precede autistic symptoms and ASD (37). In our prospective study, the timing of neuromotor assessment makes it very likely that nonoptimal performance of infants precedes the development of autistic symptoms rather than the opposite. Neuromotor development was measured as early as 9 to 20 weeks of age. Therefore, it probably preceded autistic traits because the early sensorimotor symptoms of autism, such as eye contact decline, are first exhibited from 1 to 6 months of age $(38,39)$, while behavioral signs typically emerge during the second part of the first year of life $(4,40)$. We showed that there is pleiotropy between nonoptimal neuromotor and autistic traits due to shared genetic susceptibility. This observation suggests that early sensorimotor impairment might serve as an early sign of later autistic symptoms.

We also found that $\mathrm{PRS}_{\mathrm{ASD}}$ and $\mathrm{PRS}_{\mathrm{ADHD}}$ were associated with less optimal senses and other observations (i.e., strabismus, fixation eyes, following movement eyes, hearing, sweating, and startled reactions) during infancy. The connection between sensory and perception processes of an individual with the environment is key to the execution of a motor task. Poor sensorimotor integration plays an important role in the disturbances of motor control typically seen in children with autism $(41,42)$. More than $90 \%$ of children with ASD present with abnormalities in sensory function, manifesting as hypo- or hypersensitiviy (43). This observation differentiates ASD from ADHD because children with ADHD more typically present hypersensitivity (44). It remains unclear whether hypoor hypersensitivity observed early in childhood with ASD reflects stimulus-specific mechanisms or moment-to-moment fluctuations in attention. Atypical maturation of early sensorimotor functioning may affect the integrity of developmental trajectories, although the mechanism is not fully understood $(12,13)$.

We observed effect modification by sex in the relationship between ADHD genetic susceptibility and autistic traits in the way that this association was present only in boys. PRS $_{\mathrm{ADHD}}$ are on autosomal genes, and therefore the distribution of genetic loci related to ADHD is similar in boys and girls. However, certain genetic loci could convey more vulnerability to environmental and social influences in boys than in girls. Sex differences also exist in developmental trajectories, with girls being more likely to experience an escalation of autistic traits later than boys in early and mid-adolescence $(45,46)$. Slower maturation of the fetal and postnatal brains extends the window of vulnerability and puts boys at greater risk for environmental toxins and prolonged stress than girls (47). It is also possible that autistic traits are more typically displayed by boys. Autistic traits in girls are often misclassified as being withdrawn and anxious, and such symptoms are considered part of their typical development.

We found independent effects of $P R S_{A S D}$ and $P R S_{A D H D}$ on neuromotor development as well as on SRS scores. This is in agreement with findings of recent articles by the Brainstorm Consortium and a previous study from Generation $\mathrm{R}$ that show no correlation between $\mathrm{PRS}_{\mathrm{ASD}}$ and $\mathrm{PRS}_{\mathrm{ADHD}}(48,49)$. In this sample, $\mathrm{PRS}_{\mathrm{ADHD}}$ predicted autistic traits better than $\mathrm{PRS}_{\mathrm{ASD}}$. Our results most likely reflect the sample size of the GWAS from which the PRSs are derived; the GWAS for ADHD ( $N=$ $55,374)$ is larger than that for ASD $(N=15,954)$. This will certainly affect the prediction accuracy of the scores (50). Second, in the Avon Longitudinal Study of Parents and Children, the genetic liability for ADHD was associated with ASDrelated traits (34). Third, higher $\mathrm{PRS}_{\mathrm{ADHD}}$ are associated with 
general psychopathology, suggesting that a considerable portion of the genetic variants associated with ADHD (which are captured by PRSs) is shared with other measures and reflect a nonspecific genetic liability toward broad childhood psychopathology.

\section{Strengths and Limitations}

This study has several strengths, including genotyping in a homogeneous group of children from the general population, comprehensive neurological examinations during infancy, and a prospective design and evaluations of autistic traits in childhood. Nonetheless, we faced limitations that require caution in the interpretation of our findings. First, selective nonresponse to neuromotor or autistic trait assessment could potentially be the source of bias in the analysis. However, we did not detect any differences in neuromotor measurements or autistic traits between infants with and without genetic assessment.

Second, because we were studying genetic variation in a nonclinical population, the $R^{2}$ values are small (overall motor development) or very small (senses and other observations). Therefore, if results are used in a clinical population, any interpretation should be done consciously.

Third, we employed a PRS approach to estimate individuallevel genetic propensities and predicted developmental outcomes in an independent target sample. However, genetic markers reflect only a fraction of total genetic and disease risk (51). In addition, predictive power of PRSs does not simply reflect the genetic correlation between discovery and target trait but rather depends on the genetic architecture of both traits and sample size (52).

\section{Conclusions}

Early neurological development is influenced by the interaction with environmental factors but is also largely a consequence of the maturation of the central nervous system. We found associations between genetic predisposition to ASD and ADHD and nonoptimal neuromotor development during infancy. Our findings suggest that a genetic correlation exists between infant neuromotor development and autistic symptoms, reflecting a shared genetic liability that independently affects motor development and autistic traits. This shared genetic liability or genetic correlation explains part of the observed heritability of both autistic traits and neuromotor development.

\section{ACKNOWLEDGMENTS AND DISCLOSURES}

The general design of the Generation R Study is supported by the Erasmus Medical Center Rotterdam, the Erasmus University Rotterdam, the Netherlands Organization for Health Research and Development (ZonMw "Geestkracht" programme 10.000.1003), the Netherlands Organization for Scientific Research (NOW), and the Ministry of Health, Welfare and Sport. The Generation R Study is conducted by the Erasmus Medical Center in close collaboration with the School of Law and the Faculty of Social Sciences of the Erasmus University Rotterdam, the Municipal Health Service Rotterdam area, the Rotterdam Homecare Foundation, and the Stichting Trombosedienst en Artsenlaboratorium Rijnmond. HT was supported by a grant of the Dutch Ministry of Education, Culture, and Science and the NWO (Grant No. 024.001.003, Consortium on Individual Development). A Ter Meulen grant financed by the Dutch Government and an Erasmus MundusWestern Balkans (ERAWEB) scholarship grant financed by the European Commission were awarded to FS (Grant No. 2013-2548/001-001-EMA2).
The authors report no biomedical financial interests or potential conflicts of interest.

\section{ARTICLE INFORMATION}

From the Generation R Study Group (FS, PRJ, YX, MHJH), Erasmus Medical Center Rotterdam, Rotterdam; Department of Child and Adolescent Psychiatry (FS, HT, PRJ, YX, AN, FCV), Erasmus Medical Center-Sophia Children's Hospital Rotterdam, Rotterdam; and Department of Complex Trait Genetics (PRJ), Center for Neuroscience and Cognitive Research, Amsterdam Neuroscience, Vrije Universiteit Amsterdam, Amsterdam, The Netherlands; Department of Pediatrics (FS, AG), Department of Population Health (AG), and Department of Environmental Medicine (AG), New York University School of Medicine, New York, New York; Department of Socia and Behavioral Sciences $(\mathrm{HT})$ and Department of Epidemiology (ER), Harvard T.H. Chan School of Public Health, Boston, Massachusetts; and Barcelona Institute for Global Health (SA), Universitat Pompeu Fabra, CIBER Epidemiología y Salud Pública, Barcelona, Spain.

Address correspondence to Henning Tiemeier, M.D., Department of Social and Behavioral Sciences, Harvard T.H. Chand School of Public Health, 677 Huntington Ave., Kresge Building, Room 619, Boston, MA 02115; E-mail: tiemeier@hsph.harvard.edu.

Received Dec 4, 2018; revised May 3, 2019; accepted Jun 3, 2019.

Supplementary material cited in this article is available online at https:// doi.org/10.1016/j.biopsych.2019.06.006.

\section{REFERENCES}

1. Constantino JN, Todd RD (2003): Autistic traits in the general population: A twin study. Arch Gen Psychiatry 60:524-530.

2. Cross-Disorder Group of the Psychiatric Genomics Consortium (2013): Identification of risk loci with shared effects on five major psychiatric disorders: A genome-wide analysis. Lancet 381:1371-1379.

3. Robinson EB, Koenen KC, McCormick MC, Munir K, Hallett V, Happe F, et al. (2011): Evidence that autistic traits show the same etiology in the general population and at the quantitative extremes (5\%, 2.5\%, and 1\%). Arch Gen Psychiatry 68:1113-1121.

4. Varcin KJ, Jeste SS (2017): The emergence of autism spectrum dis order: Insights gained from studies of brain and behaviour in high-risk infants. Curr Opin Psychiatry 30:85-91.

5. Lord C, Elsabbagh M, Baird G, Veenstra-Vanderweele J (2018): Autism spectrum disorder. Lancet 392:508-520.

6. Peralta V, Cuesta MJ (2017): Motor abnormalities: From neuro developmental to neurodegenerative through "functional" (neuro)psychiatric disorders. Schizophr Bull 43:956-971.

7. Fournier KA, Hass CJ, Naik SK, Lodha N, Cauraugh JH (2010): Motor coordination in autism spectrum disorders: A synthesis and metaanalysis. J Autism Dev Disord 40:1227-1240.

8. Bryson SE, Zwaigenbaum L, Brian J, Roberts W, Szatmari P Rombough V, et al. (2007): A prospective case series of high-risk infants who developed autism. J Autism Dev Disord 37:12-24.

9. Teitelbaum P, Teitelbaum O, Nye J, Fryman J, Maurer RG (1998): Movement analysis in infancy may be useful for early diagnosis of autism. Proc Natl Acad Sci U S A 95:13982-13987.

10. Serdarevic F, Ghassabian A, van Batenburg-Eddes T, White $T$, Blanken LME, Jaddoe VWV, et al. (2017): Infant muscle tone and childhood autistic traits: A longitudinal study in the general population. Autism Res 10:757-768.

11. Serdarevic F, van Batenburg-Eddes T, Mous SE, White T, Hofman A Jaddoe VW, et al. (2016): Relation of infant motor development with nonverbal intelligence, language comprehension and neuropsychological functioning in childhood: A population-based study. Dev Sci 19:790-802.

12. Denisova K, Zhao G (2017): Inflexible neurobiological signatures precede atypical development in infants at high risk for autism. Sci Rep $7: 11285$.

13. Hazlett HC, Gu H, Munsell BC, Kim SH, Styner M, Wolff JJ, et al. (2017): Early brain development in infants at high risk for autism spectrum disorder. Nature 542:348-351. 
14. Mous SE, Jiang A, Agrawal A, Constantino JN (2017): Attention and motor deficits index non-specific background liabilities that predict autism recurrence in siblings. J Neurodev Disord 9:32.

15. Kooijman MN, Kruithof CJ, van Duijn CM, Duijts L, Franco OH, van IJzendoorn MH, et al. (2016): The Generation R Study: Design and cohort update 2017. Eur J Epidemiol 31:1243-1264.

16. Serdarevic F, Jansen PR, Ghassabian A, White T, Jaddoe VWV, Posthuma D, et al. (2018): Association of genetic risk for schizophrenia and bipolar disorder with infant neuromotor development. JAMA Psychiatry 75:96-98.

17. Medina-Gomez C, Felix JF, Estrada K, Peters MJ, Herrera L, Kruithof CJ, et al. (2015): Challenges in conducting genome-wide association studies in highly admixed multi-ethnic populations: The Generation R Study. Eur J Epidemiol 30:317-330.

18. Purcell S, Neale B, Todd-Brown K, Thomas L, Ferreira MA, Bender D, et al. (2007): PLINK: A tool set for whole-genome association and population-based linkage analyses. Am J Hum Genet 81:559-575.

19. Delaneau O, Marchini J, Zagury JF (2011): A linear complexity phasing method for thousands of genomes. Nat Methods 9:179-181.

20. Howie BN, Donnelly P, Marchini J (2009): A flexible and accurate genotype imputation method for the next generation of genome-wide association studies. PLoS Genet 5:e1000529.

21. Patterson N, Price AL, Reich D (2006): Population structure and eigenanalysis. PLoS Genet 2:e190.

22. Price AL, Zaitlen NA, Reich D, Patterson N (2010): New approaches to population stratification in genome-wide association studies. Nat Rev Genet 11:459-463.

23. Euesden J, Lewis CM, O'Reilly PF (2015): PRSice: Polygenic risk score software. Bioinformatics 31:1466-1468.

24. Touwen BCL (1979): Examination of the Child With Minor Neurological Dysfunction. London: Heinemann.

25. de Groot L, Hopkins B, Touwen BC (1992): A method to assess the development of muscle power in preterms after term age. Neuropediatrics 23:172-179.

26. van Batenburg-Eddes T, de Groot L, Steegers EAP, Hofman A, Jaddoe VWV, Verhulst FC, et al. (2010): Fetal programming of infant neuromotor development: The Generation R Study. Pediatr Res $67: 132-137$.

27. Peters LHJ, Maathuis KGB, Kouw E, Hamming M, Hadders-Algra M (2008): Test-retest, inter-assessor and intra-assessor reliability of the modified Touwen examination. Eur J Paediatr Neurol 12:328-333.

28. Cross-Disorder Group of the Psychiatric Genomics Consortium, Lee SH, Ripke S, Neale BM, Faraone SV, Purcell SM, et al. (2013): Genetic relationship between five psychiatric disorders estimated from genome-wide SNPs. Nat Genet 45:984-994.

29. Halladay AK, Bishop S, Constantino JN, Daniels AM, Koenig K, Palmer K, et al. (2015): Sex and gender differences in autism spectrum disorder: Summarizing evidence gaps and identifying emerging areas of priority. Mol Autism 6:36.

30. Lee SH, Yang J, Goddard ME, Visscher PM, Wray NR (2012): Estimation of pleiotropy between complex diseases using singlenucleotide polymorphism-derived genomic relationships and restricted maximum likelihood. Bioinformatics 28:2540-2542.

31. Yang J, Benyamin B, McEvoy BP, Gordon S, Henders AK, Nyholt DR, et al. (2010): Common SNPs explain a large proportion of the heritability for human height. Nat Genet 42:565-569.

32. Martin J, Hamshere ML, Stergiakouli E, O'Donovan MC, Thapar A (2014): Genetic risk for attention-deficit/hyperactivity disorder contributes to neurodevelopmental traits in the general population. Biol Psychiatry 76:664-671.

33. Robinson EB, St Pourcain B, Anttila V, Kosmicki JA, Bulik-Sullivan B, Grove J, et al. (2016): Genetic risk for autism spectrum disorders and neuropsychiatric variation in the general population. Nat Genet 48:552-555.
34. Riglin L, Collishaw S, Thapar AK, Dalsgaard S, Langley K, Smith GD, et al. (2016): Association of genetic risk variants with attention-deficit/ hyperactivity disorder trajectories in the general population. JAMA Psychiatry 73:1285-1292.

35. Bolton PF, Golding J, Emond A, Steer CD (2012): Autism spectrum disorder and autistic traits in the Avon Longitudinal Study of Parents and Children: Precursors and early signs. J Am Acad Child Adolesc Psychiatry 51:249-260.e225.

36. Gurevitz M, Geva R, Varon M, Leitner Y (2012): Early markers in infants and toddlers for development of ADHD. J Atten Disord 18:14-22.

37. Mahajan R, Dirlikov B, Crocetti D, Mostofsky SH (2016): Motor circuit anatomy in children with autism spectrum disorder with or without attention deficit hyperactivity disorder. Autism Res 9:67-81.

38. Jones W, Klin A (2013): Attention to eyes is present but in decline in 2-6-month-old infants later diagnosed with autism. Nature 504:427-431.

39. Di Giorgio E, Frasnelli E, Rosa-Salva O, Luisa Scattoni M, Puopolo M, Tosoni D, et al. (2016): Difference in visual social predispositions between newborns at low- and high-risk for autism. Sci Rep 6:26395.

40. Ozonoff S, losif A-M, Baguio F, Cook IC, Hill MM, Hutman T, et al. (2010): A prospective study of the emergence of early behavioral signs of autism. J Am Acad Child Adolesc Psychiatry 49:256-266.e252.

41. Velasques B, Machado S, Paes F, Cunha M, Sanfim A, Budde H, et al. (2011): Sensorimotor integration and psychopathology: Motor control abnormalities related to psychiatric disorders. World J Biol Psychiatry 12:560-573.

42. Coynel D, Marrelec G, Perlbarg V, Pélégrini-Issac M, Van de Moortele P-F, Ugurbil K, et al. (2010): Dynamics of motor-related functional integration during motor sequence learning. Neurolmage 49:759-766.

43. Leekam SR, Nieto C, Libby SJ, Wing L, Gould J (2007): Describing the sensory abnormalities of children and adults with autism. J Autism Dev Disord 37:894-910.

44. Visser JC, Rommelse NN, Greven CU, Buitelaar JK (2016): Autism spectrum disorder and attention-deficit/hyperactivity disorder in early childhood: A review of unique and shared characteristics and developmental antecedents. Neurosci Biobehav Rev 65:229-263.

45. Kleberg JL, Nyström P, Bölte S, Falck-Ytter T (2019): Sex differences in social attention in infants at risk for autism. J Autism and Dev Disord 49:1342-1351.

46. Mandy W, Pellicano L, St Pourcain B, Skuse D, Heron J (2018): The development of autistic social traits across childhood and adolescence in males and females. J Child Psychol Psychiatry 59:11431151.

47. Zahn-Waxler C, Shirtcliff EA, Marceau K (2008): Disorders of childhood and adolescence: Gender and psychopathology. Annu Rev Clin Psychol 4:275-303.

48. Anttila V, Bulik-Sullivan B, Finucane HK, Walters RK, Bras J, Duncan L, et al. (2018): Analysis of shared heritability in common disorders of the brain. Science 360:eaap8757.

49. Alemany S, Jansen PR, Muetzel RL, Marques N, El Marroun H, Jaddoe VWV, et al. (2019): Common polygenic variations for psychiatric disorders and cognition in relation to brain morphology in the general pediatric population. J Am Acad Child Adolesc Psychiatry 58:600-607.

50. Martin AR, Daly MJ, Robinson EB, Hyman SE, Neale BM (2018): Predicting polygenic risk of psychiatric disorders [published online ahead of print Dec 28]. Biol Psychiatry.

51. Wray NR, Lee SH, Mehta D, Vinkhuyzen AAE, Dudbridge F, Middeldorp CM (2014): Research review: Polygenic methods and their application to psychiatric traits. J Child Psychol Psychiatry 55:10681087.

52. Dudbridge F (2013): Power and predictive accuracy of polygenic risk scores. PLoS Genet 9:e1003348. 